\title{
Implementation of a carbapenem prescribing algorithm at a community hospital
}

\author{
Stacy E. Shimata PharmD ${ }^{1}$, Christine M. Antczak PharmD ${ }^{1}$, Phillip S. Yu MD ${ }^{1}$ and Kavita K. Trivedi MD ${ }^{2}$ \\ ${ }^{1}$ Sutter Tracy Community Hospital, Tracy, California and ${ }^{2}$ Trivedi Consults, Berkeley, California
}

To the Editor-Sutter Tracy Community Hospital (STCH) is a nonprofit 79-bed acute-care hospital located in Northern California serving more than 100,000 people in the Central Valley region. In 2015, the STCH Antimicrobial Stewardship Program (ASP) established a multidisciplinary committee under the leadership of a consultant ASP physician and an ASP certified pharmacist to coordinate antibiotic stewardship efforts and to support the implementation of new initiatives.

Carbapenems have broad-spectrum activity against grampositive and gram-negative bacteria, including organisms producing extended spectrum $\beta$-lactamases (ESBLs). Unrestricted use and overuse of these agents leads to limited susceptibility profiles and reduced treatment options. Carbapenem-resistant Enterobacteriaceae (CRE) bacteria have been on the rise and have been deemed an urgent threat level by the Centers for Disease Control and Prevention (CDC). More than 9,000 healthcare-associated infections are caused by CRE bacteria each year, with a reported 40-50\% mortality rate for invasive infections. ${ }^{1,2}$ An additional serious threat is multidrug-resistant Pseudomonas aeruginosa, which accounts for $13 \%$ of all hospital-acquired $P$. aeruginosa infections. These organisms often exhibit high rates of cross resistance with all $\beta$-lactam antibiotics. ${ }^{1,3}$

Promoting the appropriate use of antibiotics by decreasing inappropriate prescribing is a key element of antibiotic stewardship to reduce the emergence of resistance. ${ }^{4}$ Studies have shown that unrestricted use of carbapenems have been linked to high rates of inappropriate use, thereby offering opportunities for the selection of narrower-spectrum agents or therapy de-escalation. Pharmacy-driven stewardship efforts have been successful with carbapenem de-escalation, but data demonstrating the direct effect of antibiotic stewardship interventions on improving their susceptibility profiles are lacking. ${ }^{5-8}$

The STCH Antimicrobial Stewardship Committee noted imipenem-cilastatin as the antipseudomonal $\beta$-lactam with the lowest susceptibility to $P$. aeruginosa (88\%) and a concerning decreasing trend from previous years (92\% in 2013). A Carbapenem Prescribing Algorithm (Fig. 1) was developed as guidance to prescribers to promote the appropriate use of carbapenems. Pharmacists utilized prospective audit and feedback for new orders that failed to meet the algorithm's use criteria and recommended alternative therapy. The objective of this stewardship initiative was to decrease overall carbapenem use by improving prescribing habits and promoting the appropriate use of these antibiotics. Buyin was obtained from hospital physicians at the Department of Medicine committee meeting prior to implementation.

Author for correspondence: Stacy E. Shimata, PharmD, Sutter Tracy Community Hospital, 1420 N Tracy Blvd, Tracy, CA 95376. Email: shimats@sutterhealth.org

Cite this article: Shimata SE, et al. (2018). Implementation of a carbapenem prescribing algorithm at a community hospital. Infection Control \& Hospital Epidemiology 2018, 39, 1503-1504. doi: 10.1017/ice.2018.256

\section{Methods}

A quasi-experimental study was conducted utilizing a retrospective medication use evaluation (MUE). Three months of pre-intervention baseline data were collected from January to March 2015. The Carbapenem Prescribing Algorithm was implemented in December 2016 with education completed with prescribers and clinical pharmacists. In addition, 3 post-intervention follow-up periods of data were collected: immediate post-intervention (January-March 2016), 6 months post-intervention (July-September 2016), and 1 year post-intervention (January-March 2017). The primary endpoints were carbapenem days of therapy adjusted per 1,000 patient days (DOT) and percent of patients who met algorithm use criteria. Secondary endpoints included antibiogram susceptibility and cost savings using pharmacy purchasing data.

\section{Results}

A 3-fold decrease in DOT was observed from baseline (131.8) to immediately post-intervention (40.2) and remained low (42.9) 6 months post-intervention. Although DOT increased to 64.5 by 1 year post-intervention, it remained $\sim 50 \%$ lower than the baseline level. At baseline, only $20 \%$ of patients met algorithm use criteria, which increased to $35 \%$ immediately post-intervention. At 6 months and 1 year post-intervention, the percentage of patients who met algorithm use criteria increased to $70 \%$ and $79 \%$ respectively, nearly 3 - to 4 -fold compared to the baseline. The overall reduction in carbapenem use improved susceptibility to $P$. aeruginosa. After implementation of the Carbapenem Prescribing Algorithm, the hospital antibiogram susceptibility increased to $89 \%$ in 2015 and to $92 \%$ in 2016 , a return to the 2013 percentage. Additionally, using 2015 as baseline, a $\$ 75,000$ pharmacy cost savings was calculated for 2016 and $\$ 65,000$ cost savings for 2017, which was attributed to the direct impact of implementing the Carbapenem Prescribing Algorithm.

\section{Discussion}

The implementation of a pharmacy-driven Carbapenem Prescribing Algorithm at a small community hospital improved prescribing habits and the appropriate use of these agents. This intervention also led to a 3 -fold reduction in overall carbapenem use, improvement of local antibiogram susceptibilities, and cost savings for the hospital. Success with the algorithm continued 6 months and 1 year post-intervention. Although carbapenem utilization increased 1 year post-intervention, $79 \%$ of this use was deemed appropriate based on algorithm criteria.

This study had some limitations. It was conducted at a small community hospital, so our results may not be generalizable to larger academic and trauma centers with varying rates of 


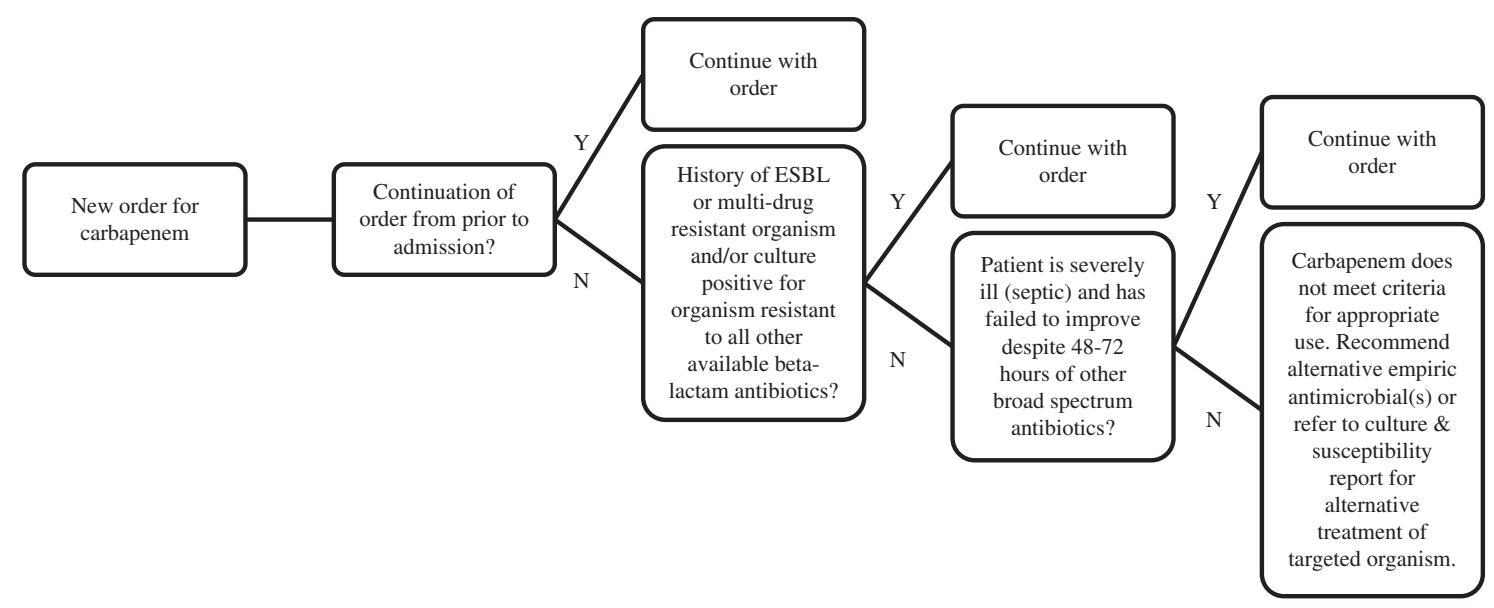

Fig. 1. Carbapenem Prescribing Algorithm

carbapenem resistance and susceptibility trends. Additionally, cost savings was calculated from antibiotic purchasing data, so these results may underestimate true cost savings. During our study period, there were several antibiotic drug shortages, so a larger supply of alternative antibiotics, such as carbapenems, may have been purchased in anticipation of a prolonged shortage.

Strengths of this study include assessment of outcomes for an extended duration, up to 1 year post-intervention, to evaluate the long- term effect of the implementation of the algorithm, which allowed study investigators to identify continued effects of the intervention. Due to a smaller physician and pharmacy personnel pool who were also amenable to the implementation of the algorithm, pharmacists were able to provide effective education on appropriate carbapenem prescribing that was sustained over time. This may also be a reason that these protocols are more successful in smaller institutions if buy-in is obtained before the protocol is implemented.

Pharmacy-driven ASP strategies may be particularly effective at both reducing antimicrobial utilization and improving antimicrobial susceptibility trends at small community hospitals.

Acknowledgments. We would like to acknowledge the STCH Antimicrobial Stewardship Committee and the Pharmacy Department for implementing the Carbapenem Prescribing Algorithm and the hospital physicians for supporting the success of our ASP.

Financial support. None reported.

Conflicts of interest. All authors report no conflicts of interest to this article.

\section{References}

1. US Department of Health and Human Services. Antimicrobial resistance threats in the United States, 2013. Centers for Disease Control and Prevention website. https://www.cdc.gov/drugresistance/threat-report2013/. Published 2013. Accessed June 12, 2018.

2. Livorsi DJ, Chorazy ML, Schweizer ML, et al. A systematic review of the epidemiology of carbapenem-resistant Enterobacteriaceae in the United States. Antimicrob Resist Infect Control 2018;55:1-9.

3. Sader HS, Huband MD, Castanheira M, et al. Pseudomonas aeruginosa antimicrobial susceptibility results from four years (2012 to 2015) of the International Network for Optimal Resistance Monitoring Program in the United States. Antimicrob Agents Chemother 2017;61:1-7.

4. Barlam TF, Cosgrove SE, Abbo LM, et al. Implementing an antibiotic stewardship program: guidelines by the Infectious Diseases Society of America and the Society for Healthcare Epidemiology of America. Clin Infect Dis 2016;62:e51-e77.

5. Lai C, Shi Z, Chen Y, et al. Effects of various antimicrobial stewardship programs on antimicrobial usage and resistance among common gramnegative bacilli causing health-care associated infections: a multicenter comparison. J Microbiol Immunol Infect 2016;49:74-82.

6. Lew KY, Ng TM, Tan M, et al. Safety and clinical outcomes of carbapenem de-escalation as part of an antimicrobial stewardship programme in an ESBL-endemic setting. J Antimicrob Chemother 2015;70:1219-1225.

7. Ahmad F, Pogue JM, Marchaim D, et al. Evaluation of the potential impact of a carbapenem de-escalation program in an academic healthcare system. $J$ Infect Public Health 2014;7:50-53.

8. Janssen J, Kinkade A, Man D. Carbapenem utilization evaluation in a large community hospital (CARBON): a quality improvement study. Can J Hosp Pharm 2015;68:327-331. 